\title{
Wireless Mesh Networks Based on MBPSO Algorithm to Improvement Throughput
}

\author{
Shivan Qasim Ameen', Firas Layth Khaleel ${ }^{2}$ \\ ${ }^{1}$ Universiti Kebangsaan Malaysia, Faculty of Information Science and Technology, Softam Department, Malaysia \\ ${ }^{2}$ Tikrit University, Faculty of Computer Science Compuer Science Department, Salah Din, Iraq
}

\begin{tabular}{l} 
Article Info \\
\hline Article history: \\
Received Dec 24, 2017 \\
Revised Mar 10, 2018 \\
Accepted Mar 24, 2018 \\
\hline
\end{tabular}

Keyword:

Heuristic algorithm MBPSO

Minimize cost of distance

Routers

Wireless mesh networks

\begin{abstract}
Wireless Mesh Networks can be regarded as a type of communication technology in mesh topology in which wireless nodes interconnect with one another. Wireless Mesh Networks depending on the semi-static configuration in different paths among nodes such as PDR, E2E delay and throughput. This study summarized different types of previous heuristic algorithms in order to adapt with proper algorithm that could solve the issue. Therefore, the main objective of this study is to determine the proper methods, approaches or algorithms that should be adapted to improve the throughput. A Modified Binary Particle Swarm Optimization (MBPSO) approach was adapted to improvements the throughput. Finally, the finding shows that throughput increased by $5.79 \%$ from the previous study.
\end{abstract}

Copyright $@ 2018$ Institute of Advanced Engineering and Science. All rights reserved.

\section{Corresponding Author:}

Firas Layth Khaleel,

Tikrit University - Faculty of Computer Science,

Computer Science Department, Salah Din, Iraq.

Email: Firas_Layth@tu.edu.iq, Firas_Layth@yahoo.com

\section{INTRODUCTION}

A mesh topology in which radio nodes are arranged, making up a communications network as shown in Figure 1, is known as a Wireless Mesh Network (WMN) [1]. This network also takes the form of an ad-hoc wireless network [2]. WMNs usually involve mesh clients, mesh routers, and gateways. The mesh clients usually comprise a variety of wireless appliances including PCs, handsets, and the like. On the other hand, the mesh routers help in traffic forwarding to and from gateways, which may not have Internet connection. The radio node coverage area that functions as one network is at times referred to as a mesh cloud and accessing this mesh cloud depends wholly on the radio nodes functioning in accord with one other to produce a radio network. A mesh network offers redundancy and is reliable [1]. Whenever a particular node stops functioning, the other nodes continue communicating with one another directly, via one intermediate node or more. Wireless Mesh Networks can self-heal and self-form [1]. Wireless Mesh Networks can be executed via several wireless technologies comprising 802.16, 802.15, and 802.11 cellular technologies and require no restriction to any protocol or technology.

The importance of Wireless mesh network leads to be used in several domains such as [1-10]. Also wireless mesh architecture is an initial phase towards offering high dynamic-bandwidth and cost-efficient networks for a particular coverage area. Excluding the cabling between nodes, a network of routers makes up the wireless mesh infrastructure. This comprises peer radio appliances that require no wiring to a cabled port, unlike traditional access points (AP) in WLAN. By separating the distances into a succession of small hops, the mesh infrastructure can convey data through large distances. Intermediate nodes help boost the signal and also cooperate in transmitting data from a particular point to another point (e.g., Point A to Point B) by making decisions for forwarding based on their understanding of the network, i.e. through implementing 
routing. This type of architecture might, with cautious design, offer an economic advantage, spectral efficiency, and high bandwidth all throughout the coverage area.

Wireless Mesh Networks have a comparatively steady topology apart from the rare malfunctioning of their nodes or added-on nodes. There are infrequent changes happening to the traffic path, as these results from the aggregation of a huge number of end users. Virtually all infrastructure mesh network traffic is either forwarded to the gateway or from it, while in client mesh networks or ad-hoc networks the flow of traffic occurs amid arbitrary node pairs [46].

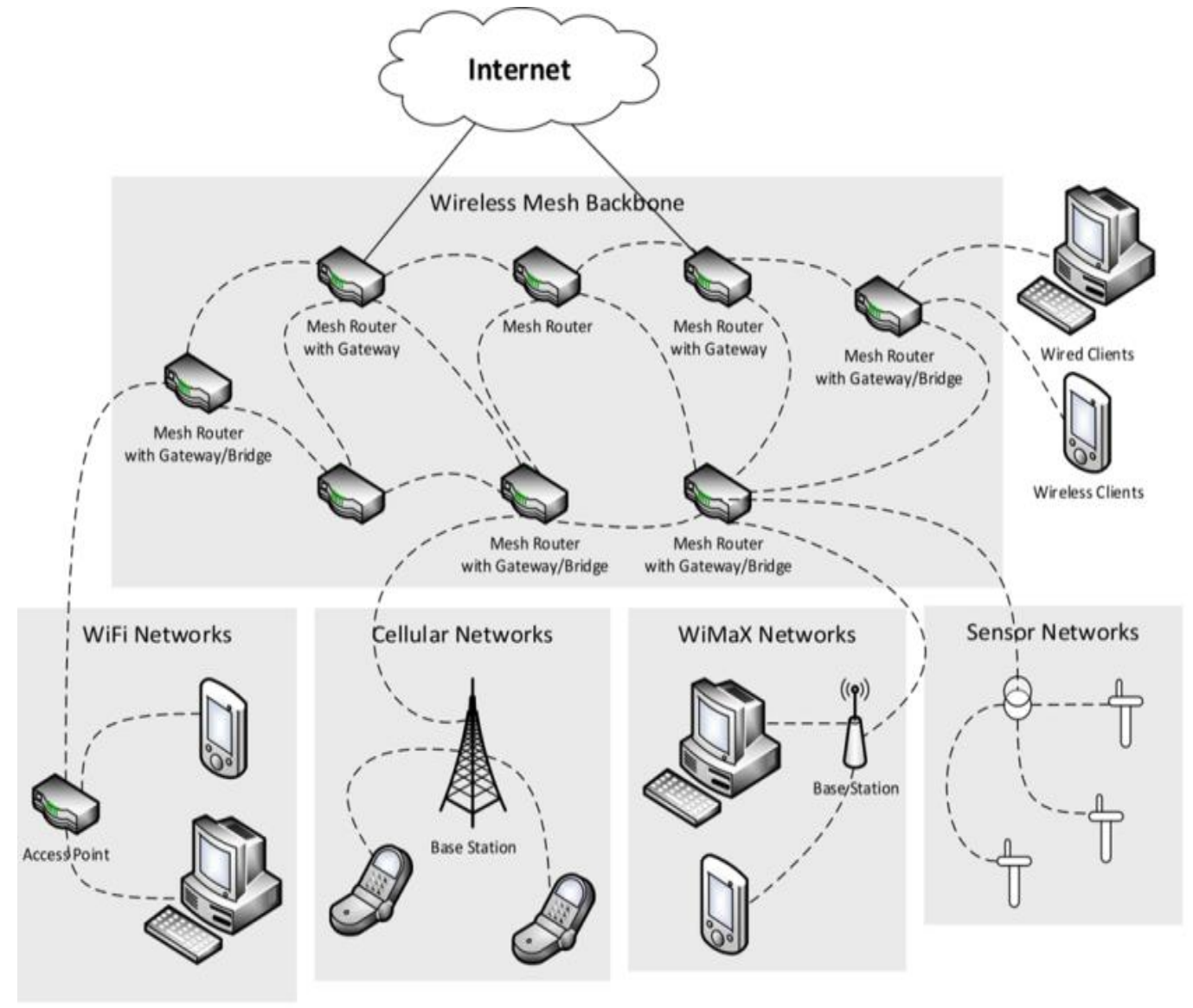

Figure 1. Wireless mesh network diagram [2]

This kind of infrastructure may be centrally handled (using a central server) or decentralized (without any central server) [47], [11] These types are comparatively low-cost and may be very resilient and reliable, as the individual node requires only the transmittance to the degree of the next node. Nodes function as routers for the transmission of data ranging from nodes that are close by to far away peers, which cannot be reached in just one hop, leading to a network that may span longer distances. A mesh network topology is also dependable, as every node is coupled to a few other nodes. Once a node falls out of the network, as a result of the failure of the hardware or any other cause, its neighbors will swiftly determine an alternative route via a routing protocol.

\section{RESEARCH BACKGROUND}

Wireless Mesh Networks can be regarded as a type of communication technology in mesh topology in which wireless nodes interconnect with one another [12], [13]. Mesh network communication tools are commonly grouped as routers, gateways, and clients. In these networks, every gateway might directly offer a type of service, and the data, which flows amid gateways and subscribers, are relayed via routers. IEEE 802.11, IEEE 802.15, and IEEE 802.16 are some of the technologies in which the networking of wireless mesh finds its application and are presumed to be the provider carrier for the problem design. When 
considering the success recorded by Wi-Fi technology (IEEE 802.11) in the substitution of wired network computers from offices and households, the main objective of this research and industry is to harness resources towards eradicating the cost of setting up and maintaining cable use in metropolitan broadband network areas. This research resulted in the enhancement of IEEE 802.16, which now performs the function of a backhaul for broadband wireless access for (WMAN) metropolitan wireless network areas [14]. The interoperable application of the IEEE 802.16 wireless family is popularly known as WiMAX [15]. The IEEE Standards Board initiated a functioning group in 1999 to formulate standards for the WMAN broadband. The founded group made available their IEEE 802.16 initial draft in February 2004. In this phase, the (SS) subscriber stations and (BS) base stations ought to be immobile and in line-of-sight, respectively [12], [16].

Another substantial improvement occurred with the IEEE 802.16e-2005 introduction, which deals with communication and mobility that are non-line-of-sight for the subscribers between BS and SS, utilization of Scalable Orthogonal Frequency Division Multiple Access, enhanced service quality support, and much more [16]. Practical problems still exist even with the level of development recorded such as the requirement for access of uneven traffic distribution in densely populated areas, the signal-to-noise ratio occurring at the edge of the cell, and coverage holes that emerge as a result of non-light-of-sight networks and shadowing, etc. The WiMAX protocols have to guarantee dependability, address coverage holes, and support utmost mobility to compete with wired broadband providers and 3G. Each challenge is in contrast to the other. Reliability decreases as rate of data increases. However, coverage area (i.e. the size of the cell) reduces as the reliability of service increases. Reduction of the size of the cell would result in an increase in the quantity of BSs for a specific area coverage, which will result in the rise of network costs [12], [16].

Relay station (RS) insertion between the BSs and SSs serves as the best solution at this point, which will route data in between the stations. The relay is utilized for the extension of network coverage range and capacity, which also connects the coverage holes e.g., shadows of buildings, therefore, enhancing end-to-end communication quality [17]. IEEE $802.16 \mathrm{j}$ is a modified version of IEEE 802.16e. It projects that data amid a SS and BS can be relayed through a RS via MMR (mobile multi-hop relay network), which utilizes the strengths of wireless multi-hop connectivity [18]. The range of coverage and quantity of Wimax is expanded with the introduction of IEEE $802.16 \mathrm{j}$, addressing the problem of building coverage holes, and thus simplifying the extension of coverage temporarily to areas with a high-density population. The architecture of the network presents several complications within the previously challenged radio access networks that provide support for mobility [19], [20] e.g., the scheduling of channel access regarding frequency reuse, RS and BS placement, resource (time and frequency) allocation, frequency and time, etc. [12], [16]. The subsequent subsections provide samples of various researches carried out on the networks of IEEE 802.16j to develop a greater level of information regarding networks and problems encountered in wireless network design, which share some similarity to the problem highlighted in this study.

In wireless communication, interference may arise due to sharing of communication medium within the stations. To solve this problem, network resources [21] suggest a scheduling algorithm, which will support spatial reuse gains from the two hops in a network that is relay-enabled. Ibrahim et. al. and Ge et. al. discovered that during their study of an analytical model to examine the capacity of a cell with the extension of a two-hop coverage, that spatial reuse could reduce losses in capacity [22], [23]. Moreover, many researches have focused on wireless network locational design. Also, a good amount of research has been done involving various wireless network providers and the topological and architectural planning for the networks. [24], [25] used an integer programming model including many algorithms based on Tabu Search and Greedy [26] to decide better positions to cover various traffic concentrations using BSs [24], [25]. The major challenge faced by the mobile industry is the migration from $2 \mathrm{G}$ to $3 \mathrm{G}$ networks in a way that satisfaction of the customer is achieved with a low cost of operation, and the number of cells that is used is also reduced to the minimum. Kaur et. al. proposed the determination of cell sites using a heuristic algorithm, which works by ranking cells from the generated simulated data and cell removal from the periodically simulated model [27].

A preliminary study was conducted by Sinha et. al. and Doppler et. al. in regard to the IEEE $802.16 \mathrm{j}$ design structure [28], [29]. One previous work designed a programming model for integers on IEEE 802.16d networks and suggested solving the issues of creating the lowest cost backhaul wireless network using heuristic algorithms (with BSs) to fulfill the SS requirements with no effect on the capacity limits in BSs [30]. A number of case studies have been presented to determine relay optimal location and to push the system output to the max within the BS-RS cell coverage in $802.16 \mathrm{j}$ networks [22]. However, these works fail to talk about the issues associated with multiple-relay planning.

Another study developed heuristic-based RSs to address the issue of network deployment and reuse of radio resouce when IEEE 802.16j MMR networks are involved [31]. Yet another study assessed the ability of a network for IEEE 802.16j via cooperative diversity for uplink transmissions [32]. The result from the 
research can be employed in analyzing the pros and cons of capacity improvement relay and deployment cost.

Vasishta et. al. discussed a programming formulation for integers in positioning BS and RS with the aim of reducing the cost of establishment under the limitation of user traffic demand [33]. Bound techniques and a standard branch were applied in solving this problem. However, unlike the case of small instances, when it comes to solving metropolitan-scale large instances, this approach is limited. In one study, a clustering approach was used on a similar problem [33]. To achieve the least amount of RSs in an MMR network, Chang et. al. recommended a heuristic algorithm [34] .

A model for relay-centric hierarchal optimization, which can be used for both optimization of network planning for RSs and radio resource MMR networks management, was also proposed [35]. The aims of the research are to make the most of the utilization of the RSs and achieve optimum reserved bandwidth. In a new algorithm formulation of the optimization issue, the problems of assignment constrained by chance are focused on, so as to achieve the ideal decisions on relay positioning and base station selection.

Shim et. al. produced a paper on the use of IEEE 802.16j technology for the enhancement of infrastructure communication in vehicular wireless networks [36]. They presumed the vehicular SSs location as known, and used the detail obtained for the ideal placement of RSs in a way that the end-to-end capability was taken full advantage of. The study combined a model of highway mobility and a nonlinear optimization model for the problem. The model solution guarantees ultimate end-to-end capabilities for SSs.

\section{THE ADVANTAGES AND DISADVANTAGES OF PREVIOUS ALGORITHMS}

In Table 1, a summary of different algorithms based on the advantage and disadvantage of each one is presented. Finally, the previous section summarizes the algorithms, methods and approaches in this field as shown in Table 1. At the same time, ideal learning environments can be created. In other words, the Heuristic algorithm for the Wireless Mesh Network has to adapt the PSO approach because this approach is more comprehensive than other algorithm functionalities. Also, a wide range of continuous optimization problems can be addressed via the successful application of PSO.

Table 1. Particle Swarm Optimization Parameters and Chosen Values

\begin{tabular}{|c|c|c|c|}
\hline No. & Algorithm & Advantages & Disadvantages \\
\hline 1 & $\begin{array}{l}\text { Heuristic } \\
\text { Algorithm [37, } \\
38]\end{array}$ & $\begin{array}{l}\text { The performance of the proposed scheme is not } \\
\text { only close to the optimal multi-path solution, it } \\
\text { also outperforms existing multi-path routing } \\
\text { schemes. }\end{array}$ & $\begin{array}{l}\text { It does not follow a standard mathematical model. } \\
\text { Lower capability for generalization. }\end{array}$ \\
\hline 2 & $\begin{array}{l}\text { Genetic } \\
\text { Algorithm [39, } \\
40]\end{array}$ & $\begin{array}{l}\text { Every routing session concurrency transmission } \\
\text { is effectively maximized through elimination of } \\
\text { interference between wireless mesh routers, } \\
\text { using this algorithm. }\end{array}$ & $\begin{array}{l}\text { To solve the problem of mesh router node } \\
\text { placement, Tabu Search, an example of a local } \\
\text { search method, and Genetic algorithms, which are } \\
\text { population-based methods, must be hybridized. }\end{array}$ \\
\hline 3 & $\begin{array}{l}\text { Adaptive Mixed } \\
\text { Bias (AMB) } \\
\text { Algorithm [41] }\end{array}$ & $\begin{array}{l}\text { Better performance is observed with the use of } \\
\text { the proposed approach concerning Adaptive } \\
\text { Mixed Bias compared to both existing mixed } \\
\text { bias approaches and IEEE } 802.11 \text { MAC. }\end{array}$ & $\begin{array}{l}\text { Varied packet rates, diverse network topologies, } \\
\text { and numerous sources through experiments must } \\
\text { be used to achieve the changes in performance and } \\
\text { in enhancing robust solutions } \\
\text { It is vital to study the parameters of Tabu Search } \\
\text { for more information to enhance the Adaptive } \\
\text { Mixed Bias method performance. }\end{array}$ \\
\hline 4 & Greedy Algorithm & $\begin{array}{l}\text { Greedy Algorithms mostly (but not always) fail } \\
\text { to find globally optimal solutions, because they } \\
\text { usually do not operate exhaustively on all the } \\
\text { data. This method can result in the prevention of } \\
\text { arriving at the best overall solution in the future, } \\
\text { as this method can commit too early to certain } \\
\text { choices. }\end{array}$ & $\begin{array}{l}\text { For instance, the Greedy Algorithms, which are } \\
\text { considered Greedy typically, fail to discover the } \\
\text { problem of graph coloring and that of the globally } \\
\text { optimum, but other NP-complete problems } \\
\text { provide a solution. Nevertheless, they function } \\
\text { exhaustively because they are swift in reaching } \\
\text { optimum approximations. }\end{array}$ \\
\hline 5 & $\begin{array}{l}\text { Local search } \\
\text { algorithm }\end{array}$ & $\begin{array}{l}\text { Generally, all local search algorithms yield } \\
\text { results that are better than the Greedy } \\
\text { Algorithms. }\end{array}$ & $\begin{array}{l}\text { However, the drawback of this improvement is a } \\
\text { longer running time. }\end{array}$ \\
\hline 6 & $\begin{array}{l}\text { Variable } \\
\text { Neighborhood } \\
\text { Search } \\
\text { Algorithms }\end{array}$ & $\begin{array}{l}\text { Local search procedure, in determining the } \\
\text { solutions to different optimization problems, is } \\
\text { very effective. }\end{array}$ & However, it can get stuck in a local minima. \\
\hline 7 & $\begin{array}{l}\text { Particle Swarm } \\
\text { Optimization }\end{array}$ & $\begin{array}{l}\text { Wide-ranging problems of optimization that are } \\
\text { continuous can apply PSO and obtain successful } \\
\text { results. }\end{array}$ & $\begin{array}{l}\text { Rarely in solving the issues, do the mesh router } \\
\text { nodes face placement problems. }\end{array}$ \\
\hline
\end{tabular}




\section{RESEARCH DESIGN}

The current research design has four phases of methodology in relation to this study the Analysis phase, Compilation phase, Innovation phase, and Validation phase are shown. At each phase, there are steps to be concluded before the succeeding phase can proceed. For example, the Analysis phase entails the stages of analysis in this study while the Innovation phase entails the enhancement and design phases, and finally, the Validation phase entails the evaluation and application stages. The following section describes in detail each of these stages.

\subsection{Analysis Phase}

This method uses the results from previous research to assess the current problem. Different research approaches, and the result quality is acquired to provide a solution to: 1) the problem of Unsplittable Flow; 2) the problem of Bin Packing; 3) the problem of Capacitated Set Covering; and 4) the Problem of Set Covering.

\subsection{Comparison Phase}

This phase involves a comparative study to determine the best method to be applied in this work. This is done through initiating a comparative study between the previous algorithms and the current methods used in this study.

\subsection{Innovation Phase}

The innovation phase considers the process of the proposed MBPSO algorithm, as follows:

a. Particle Swarm Optimization (PSO)

b. Binary Particle Swarm Optimization (BPSO)

c. Modified Binary Particle Swarm Optimization (MBPSO)

\subsection{Evaluation Phase}

To ensure that the algorithm works correctly, the validation phase goes through three metrics after the Heuristic algorithm is completed. These metrics are throughput, End-to-End Delay (E2E DELAY), and Packet Delivery Ratio (PDR). Finally, the result is compared with the nearest technical study [42] in order to measure the results improvement from the original one.

\section{PERFORMANCE METRICS}

Two basic performance metrics such as the ones that run via E2E delay and packet delivery fraction have been proposed in numerous works [43], [44]. Additionally, simulation is considered with the mobility pattern of nodes. To achieve delay and packet drop, Mirjalili et. al. propose the use of a random waypoint mobility model [45].

\subsection{Packet Delivery Ratio (PDR)}

The number of delivered packets is divided by the destination to give PDR. To calculate PDR and to determine the loss rate of the packet, the number of packets given by the application layer of the source is used to divide the number of packets received by the destination. In this way, the maximum network throughput becomes limited. In the routing protocol, an imperative factor to be accomplished is PDR, as there is no margin for error in a real-life environment like flooding and earthquakes.

\subsection{End-to-End Delay (E2E DELAY)}

The data packet will arrive at the endpoint within the time that is averaged out. For the metric calculation, the arrival time of the first data packet is used to subtract the time at which the first packet was transmitted.

\subsection{Throughput}

The average ratio of the total simulation time duration to the successful data packets is the average throughput metric. The unit of Kilobits per second (Kbytes/sec) is used to measure average throughput, where the efficiency and effectiveness of the routing protocol in receiving data packets by destination is measured. 


\section{EVALUATION RESULT}

Cost optimization is an area of research in WMNs. Our research is based upon improving the solution proposed by [42] Khaled and Shah Mostafa [42]. The author considered cost optimization without taking the distance between nodes into consideration. Our research therefore updated the optimization function to take into consideration the distances between the different nodes, using the Modified Binary Particle Swarm Optimization (MBPSO) approach. The results are positive and our approach shows noticeable improvement compared to the benchmark study. The PDR shows an approximate increase of $22.47 \%$, whereas the E2E delay saw an approximate decrease of $21.14 \%$, and finally the throughput increased by $5.79 \%$ from the previous work as shown in Figure 2.

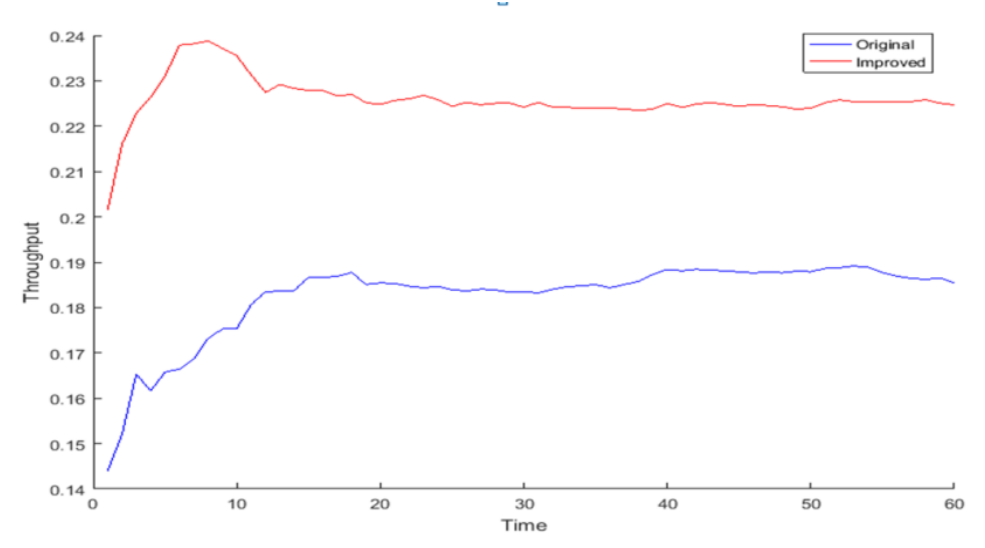

Figure 2. Throughput comparisons between the original and modified objective function

\section{DISCUSSION AND CONCLUSION}

The main contribution of this study is the proposed proper MBPSO algorithms used in this study to solve the research problem. This approach is adapted from previous work, so the main point of this study is to compare the result of this study with previous work. This work assumes the same coverage area for all nodes. Further development could be done by considering different coverage areas according to node energy and node priority. In addition, testing the performance on a range of node speeds would be a more practical scenario, with considering the speed as an influence in the standard function. Furthermore, some nodes in the network might not be trusted to transport the packet.

These nodes have a nature of selfishness, so there should be a criterion to detect and avoid them. Future works should cover the aspects of different ranges of coverage zone, speeds, and confidence for the nodes of the network. BPSO is simple and highly robust. The BPSO can solve multidimensional and multimodal optimization problems because this algorithm uses control parameters that are simple. Multidimensional functional optimization problems can also be addressed with the use of the BPSO algorithm. However, this issue is beyond the scope of the current study.

There are several directions in future work that could be implemented to enhance the performance metrics used in this study. For instance, it is possible to consider other network metrics such as packet size, number of nodes, and simulation time. This can increase the practicality and efficiency of the algorithm for functioning in the real world. On the other hand, future works on the MBPSO algorithm should probably concentrate on a particular part of the MBPSO algorithm, which could be expected to provide good cost minimization among node connections.

The research problem is addressed using heuristics that are based on different variations of heuristic algorithms. This study tested the algorithms based on 500-5000-sized nodes. Finally, this study compared the algorithm results obtained with previous work. The finding shows that PDR shows an approximate increase of $22.47 \%$, whereas the E2E delay saw an approximate decrease of $21.14 \%$, and finally the throughput increased by $5.79 \%$ from the benchmark study.

\section{CONCLUSION}

Cost optimization is an area of research with regard to WMNs. This research was based upon improving the solution proposed by Nleya and Sindiso M. [15] in this regard. They have considered cost optimization without taking distance between nodes into consideration. This research considered the problem 
using a Modified Binary Particle Swarm Optimization (MBPSO) approach by updating the optimization function to take into consideration the distances between the different nodes. The results were positive and this approach showed noticeable improvement compared to the benchmark. The PDR showed an approximate increase of $17.83 \%$ whereas the E2E delay saw an approximate decrease of $8.33 \%$, and finally the throughput increased by $5.79 \%$ from the benchmark study.

\section{ACKNOWLEDGEMENT}

This work is supported by Ministry of Education of Malaysia, Grant no: FRGS/1/2015/ICT04/UKM/02/3

\section{REFERENCES}

[1] Ameen, S.Q. and Muniyandi, R.C., 2017. Improvement at Network Planning using Heuristic Algorithm to Minimize Cost of Distance between Nodes in Wireless Mesh Networks. International Journal of Electrical and Computer Engineering (IJECE), 7(1), pp.309-315.

[2] Toh, Chai K .2001. Ad hoc mobile wireless networks: protocols and systems, Pearson Education.

[3] F.L. Khaleel, N.S. Ashaari, T.S. Meriam, T. Wook, and A. Ismail, "Programming Learning Requirements Based on Multi Perspectives", International Journal of Electrical and Computer Engineering, vol. 7, pp. 1-8., 2017.

[4] F.L. Khaleel, T.S.M.T. Wook, N.S. Ashaari, and A. Ismail, "Gamification Elements for Learning Applications", International Journal on Advanced Science, Engineering and Information Technology, in press, vol. 6, pp. 868-874, 2016 .

[5] F.L. Khaleel, N.S. Ashaari, T.S. Meriam, T. Wook, and A. Ismail, "The Architecture of Dynamic Gamification Elements Based Learning Content", Journal of Convergence Information Technology, vol. 11, pp. 164-177, $2016 \mathrm{a}$.

[6] F.L. Khaleel, N.S. Ashaari, T.S. Meriam, T. Wook, and A. Ismail, "User-Enjoyable Learning Environment Based on Gamification Elements", in International Conference on Computer, Communication, and Control Technology (I4CT 2015),, Kuching, Sarawak, Malaysia, 2015b, p. 221.

[7] F.L. Khaleel, N.S. Ashaari, T.S. Meriam, T. Wook, and A. Ismail, "The study of gamification application architecture for programming language course", in Proceedings of the 9th International Conference on Ubiquitous Information Management and Communication, 2015a, p. 17.

[8] F.L. Khaleel, "Recruitment and Job Search Application", Universiti Utara Malaysia, 2011.

[9] F.L. Khaleel, N.S. Ashaari, T.S. Meriam, T. Wook, and A. Ismail, "Gamification-Based Learning Framework for a Programming Language", in International Conference on Electrical Engineering and Informatics (ICEEI 2017),Langkawi, Kedah, Malaysia, 2018, In Press.

[10] F.L. Khaleel, N.S. Ashaari, T.S. Meriam, T. Wook, and A. Ismail, "Methodology for Developing GamificationBased Learning Programming Language Framework", in International Conference on Electrical Engineering and Informatics (ICEEI 2017),Langkawi, Kedah, Malaysia, 2018, In Press.

[11] Cheng, Ho Ting and Weihua Zhuang .2009b. QoS-driven node cooperative resource allocation for wireless mesh networks with service differentiation. Global Telecommunications Conference, GLOBECOM 2009, IEEE.

[12] Gupta, Bhupendra Kumar, Patnaik, Srikanta, Mallick, Manas Kumar, \& Nayak, Ajit Kumar. (2017). Dynamic routing algorithm in wireless mesh network. International Journal of Grid and Utility Computing, 8(1), 53-60.

[13] Akyildiz, Ian F, Xudong Wang and Weilin Wang. 2005. "Wireless mesh networks: a survey". Computer networks 47.4: 445-487.

[14] Zenaldan, Feras, Hassan, Suhaidi, \& Habbal, Adib. (2017). Vertical Handover in Wireless Heterogeneous Networks. Journal of Telecommunication, Electronic and Computer Engineering (JTEC), 9(1-2), 81-85.

[15] Nleya, Sindiso M. (2016). Design and optimisation of a low cost Cognitive Mesh Network. University of Cape Town.

[16] Saini, Jatinder Singh, \& Sohi, Balwinder Singh. (2016). A Survey on Channel Assignment Techniques of MultiRadio Multi-channel Wireless Mesh Network. Indian Journal of Science and Technology, 9(42).

[17] Dua, Amit. (2016). Efficient Data Dissemination in Vehicular Ad Hoc Networks. THAPAR UNIVERSITY, PATIALA

[18] Malm, Nicolas. (2016). Ultra-reliable Network-controlled D2D.

[19] Shaukat, Usman, Ahmed, Ejaz, Anwar, Zahid, \& Xia, Feng. (2016). Cloudlet deployment in local wireless networks: Motivation, architectures, applications, and open challenges. Journal of Network and Computer Applications, 62, 18-40.

[20] Bellalta, Boris, Bononi, Luciano, Bruno, Raffaele, \& Kassler, Andreas. (2016). Next generation IEEE 802.11 Wireless Local Area Networks: Current status, future directions and open challenges. Computer Communications, $75,1-25$.

[21] Meng, Tong, Wu, Fan, Yang, Zheng, Chen, Guihai, \& Vasilakos, Athanasios V. (2016). Spatial reusability-aware routing in multi-hop wireless networks. IEEE Transactions on Computers, 65(1), 244-255.

[22] Ibrahim, Jawwad, Rehman, A, Ilyas, M Saad Bin, Shehzad, Mohsin, \& Ashraf, Maryum. (2016). Optimization and Traffic Management in IEEE 802.16 Multi-hop Relay Stations using Genetic and Priority Algorithms. International Journal of Computer Science and Information Security, 14(7), 599. 
[23] Ge, Xiaohu, Tu, Song, Mao, Guoqiang, Wang, Cheng-Xiang, \& Han, Tao. (2016). 5G ultra-dense cellular networks. IEEE Wireless Communications, 23(1), 72-79.

[24] Zeng, Xiaoping, Sun, Meng, Jian, Xin, Du, Derong, \& Miao, Lijuan. (2017). Optimal base stations planning for Coordinated Multi-Point system. AEU-International Journal of Electronics and Communications, 73, 193-201.

[25] Goudos, Sotirios K, Deruyck, Margot, Plets, David, Martens, Luc, \& Joseph, Wout. (2017). Optimization of Power Consumption in 4G LTE Networks Using a Novel Barebones Self-adaptive Differential Evolution Algorithm. Telecommunication Systems, 1-12.

[26] HA, Mahmoud Pesaran, Huy, Phung Dang, \& Ramachandaramurthy, Vigna K. (2016). A review of the optimal allocation of distributed generation: Objectives, constraints, methods, and algorithms. Renewable and Sustainable Energy Reviews.

[27] Kaur, Ravneet, \& Kumar, Ashwani. (2016). An Approach for Selecting Optimum Number of Base Stations and Optimizing Site Locations using Flower Pollination Algorithm. International Journal of Computer Applications, 133(10), 34-39.

[28] Sinha, Koushik, Ghosh, Sasthi C, \& Sinha, Bhabani P. (2016). Wireless Networks and Mobile Computing: CRC Press.

[29] Doppler, Klaus, Redana, Simone, Wódczak, Michał, Rost, Peter, \& Wichman, Risto. (2009). Dynamic resource assignment and cooperative relaying in cellular networks: Concept and performance assessment. EURASIP Journal on Wireless Communications and Networking, 2009(1), 475281.

[30] Chen, Chi-Yuan, Tseng, Fan-Hsun, Lai, Chin-Feng, \& Chao, Han-Chieh. (2015). Network planning for mobile multi-hop relay networks. Wireless Communications and Mobile Computing, 15(7), 1142-1154.

[31] Murugadass, Arthi, \& Pachiyappan, Arulmozhivarman. (2017). Fuzzy Logic Based Coverage and Cost Effective Placement of Serving Nodes for 4G and Beyond Cellular Networks. Wireless Communications and Mobile Computing, 2017.

[32] Wang, Yupeng, Su, Xin, Choi, Dongmin, \& Choi, Chang. (2016). Coordinated Scheduling Algorithm for System Utility Maximization With Heterogeneous QoS Requirements in Wireless Relay Networks. IEEE Access, 4, 83518361.

[33] Vasishta, Anuj, Gzara, Fatma, Ho, Pin-Han, \& Lin, Bin. (2016). Optimal location planning of relay-based next generation wireless access networks. Wireless Networks, 22(7), 2159-2172.

[34] Chang, Jau-Yang, \& Chen, Yun-Wei. (2016). A relay station deployment scheme with a rotational clustering algorithm for multi-hop relay networks. Paper presented at the System Science and Engineering (ICSSE), 2016 International Conference on.

[35] Yan, Yang, Huang, Jianwei, \& Wang, Jing. (2013). Dynamic bargaining for relay-based cooperative spectrum sharing. IEEE Journal on Selected Areas in Communications, 31(8), 1480-1493.

[36] Shim, Kyusung, Do, Nhu Tri, \& An, Beongku. (2017). Performance Analysis of Physical Layer Security of Opportunistic Scheduling in Multiuser Multirelay Cooperative Networks. Sensors, 17(2), 377.

[37] Matam, Rakesh and Somanath Tripathy. 2013. "Improved heuristics for multicast routing in wireless mesh networks". Wireless networks 19.8: 1829-1837.

[38] Ling, Song, Cao Jie and Yang Xue-jun .2010. Multi-path anycast routing based on ant colony optimization in multi-gateway WMN. 2010 5th International Conference on Computer Science and Education. ICCSE, IEEE.

[39] Jia, Jie, et al. .2012. Traffic aware resource allocation for throughput optimization in cognitive radio wireless mesh networks. 2012 7th International Symposium on Wireless and Pervasive Computing. ISWPC, IEEE.

[40] Xhafa, Fatos, Admir Barolli and Makoto Takizawa .2011. A tabu search algorithm for efficient node placement in wireless mesh networks. 2011 Third International Conference on Intelligent Networking and Collaborative Systems .INCoS, IEEE.

[41] Ernst, Jason B. and Thabo Nkwe .2010. Adaptive mixed bias resource allocation for wireless mesh networks. 2010 International Conference on Broadband, Wireless Computing, Communication and Applications. BWCCA, IEEE.

[42] Khaled, Shah Mostafa 2012. Heuristic algorithms for wireless mesh network planning, Diss. Lethbridge, Alta.: University of Lethbridge, Dept. of Mathematics and Computer Science, 2012.

[43] Paschos, Georgios S, Petteri Mannersalo and Thomas Michael Bohnert. 2008. Cell capacity for ieee 802.16 coverage extension. Consumer Communications and Networking Conference, 2008. CCNC 2008. 5th IEEE, IEEE.

[44] Lin, Hua, Santhoshkumar Sambamoorthy, Sandeep Shukla, James Thorp and Lamine Mili .2011. Power system and communication network co-simulation for smart grid applications. Innovative Smart Grid Technologies. ISGT, 2011 IEEE PES, IEEE.

[45] Mirjalili, Seyedali, \& Lewis, Andrew. (2013). S-shaped versus V-shaped transfer functions for binary particle swarm optimization. Swarm and Evolutionary Computation, 9, 1-14.

[46] Mirjalili, Seyedali, \& Lewis, Andrew. (2013). S-shaped versus V-shaped transfer functions for binary particle swarm optimization. Swarm and Evolutionary Computation, 9, 1-14.

[47] Jun, Jangeun and Mihail L Sichitiu. 2003. "The nominal capacity of wireless mesh networks". IEEE wireless communications. 10.5: 8-14.

[48] Cheng, Ho Ting and Weihua Zhuang. 2009a. "QoS-driven MAC-layer resource allocation for wireless mesh networks with non-altruistic node cooperation and service differentiation". IEEE Transactions on Wireless Communications. 8.12: 6089-6103. 